\title{
Tecnologías de la información y comunicación en el proceso de adquisición de la lectoescritura ${ }^{1}$
}

\author{
Patricia Droguett Tidy² y Margarita Aravena Gaete ${ }^{3}$
}

Recibido 26 de octubre 2018 - Aceptado 28 de noviembre 2018

\begin{abstract}
RESUMEN
El artículo describe los resultados obtenidos en la investigación que buscó evaluar el uso de las TIC en el proceso de adquisición de la lectoescritura. El objetivo fue identificar en qué medida el uso de las tecnologías de información y comunicación constituyen un aporte en el proceso de enseñanza aprendizaje de la lectura y escritura en niños y niñas de primer año de educación primaria. Se llevó a cabo bajo el paradigma interpretativo, con enfoque cualitativo. Se realizó en un colegio particular pagado, con un total de I.050 alumnos. La investigación tomó como muestra dos niveles de primer año de primaria. En uno de ellos se constató que la profesora incorporaba el uso de las TIC a su práctica docente; el curso paralelo, por su parte, no incluyó el uso de recursos tecnológicos con fines educativos. La recogida de información se realizó mediante entrevistas a docentes, notas de campo y encuestas. Se concluye que existe una influencia positiva al incorporar el uso de las TIC, siempre que se realice de manera sistemática, planificada y como apoyo al docente. Para ello resulta indispensable que la institución educativa provea los recursos y la capacitación necesaria.
\end{abstract}

Palabras clave: comunicación, escritura, educación primaria, lectura, tecnologías de la información.

\section{Information and communication technologies in the literacy process}

\section{ABSTRACT}

This article describes the results obtained in a research to evaluate the use of ICT in the process of literacy. The objective was to identify the extent to which the use of information and communication technologies is a contribution in the reading and writing teaching and learning process in children at first grade of primary education. It was carried out under an interpretive paradigm with a qualitative approach and applied in a paid private school, with a total of 1050 students. The research was based on two levels of first grade of primary school. In one of them, it found that the teacher incorporated the use of ICT

I Tesis para obtener el grado de Magíster en Educación, mención Didáctica para las Matemáticas y Lenguaje.

2 Magíster en Educación, Universidad Andrés Bello (UNAB). Profesora Colegio Dunalastair Peñalolén, Chile, Academic support from level Playgroup to Fourth Grade, chilena. E-mail: pdroguett@dunalastair.cl

3 Doctora en Educación, Universidad Alcalá de Hénares. Profesora invitada Universidad Autónoma de Barcelona, España, chilena. E-mail: margarita.aravena@uab.cat 
into her teaching practice; but the other course did not include technological resources for education purposes. The information was collected through the following techniques: Interviewing teachers, field notes and survey. In conclusion, there is a positive influence when incorporating the use of ICTs, provided that this is done in a systematic and planned manner and as a support for teachers. Therefore, it is essential that the education institution provide the necessary and relevant resources and training.

Keywords: Communication, writing, primary education, reading, information technology

\section{Introducción}

El incesante desarrollo tecnológico en la comunicación ha generado un cambio importante en nuestros tiempos, lo que obliga a los responsables de delinear los estamentos educativos a adaptarse y renovarse, para proveer a los futuros hombres y mujeres de la sociedad del siglo XXI las destrezas que les permitirán avanzar en el crecimiento de la humanidad (García-Rodríguez \& Gómez Díaz, 2017). Para ello, se piensa que es indispensable el uso de las herramientas tecnológicas adecuadas a los requerimientos de los distintos niveles educativos por parte de los principales agentes del proceso de enseñanza aprendizaje: profesores y alumnos. ¿Qué son estas herramientas tecnológicas?.

Falieres (2006), denomina:

[...]TIC a las tecnologías de la información y la comunicación, es decir, al conjunto de tecnologías que permiten la adquisición, producción y almacenamiento, tratamiento, comunicación, registro y presentación de informaciones, en forma de voz, imágenes y datos contenidos en señales de naturaleza acústica, óptica o electromagnética. (p. 26I).

En Chile, conscientes de la importancia de incorporar la realidad tecnológica a la educación, el Ministerio de Educación creó en I992 el programa Enlaces, que busca aportar al mejoramiento de la calidad de la educación por medio de la informática educativa, promoviendo el desarrollo de una cultura digital. Actúa en todos los establecimientos educacionales subvencionados de Chile. Una de sus prioridades es integrar el uso de las TIC al trabajo que realiza el docente en aula, con el fin de estrechar la brecha digital en sectores 
más vulnerables, dando la oportunidad a un mayor número de niños de desarrollar habilidades del siglo XXI.

Bien se sabe que un hito importante en la vida de todo ser humano es el momento en que se adquieren las habilidades de lectoescritura; el lenguaje, en toda su expresión, permite al ser humano construir y entender su realidad, y da acceso a nuevos conocimientos $y$ perspectivas de crecimiento intelectual.

La presente investigación buscó dilucidar si la incorporación de las TIC en el proceso de adquisición de las habilidades de lectura y escritura marca una diferencia positiva. La incorporación de las tecnologías al campo educativo exige no solo un esfuerzo económico, sino también el compromiso de toda la comunidad pedagógica para capacitarse y hacer de estas herramientas tecnológicas un real aporte. Hoy, en Chile, las TIC se incorporan al currículo mediante el uso de internet -como un medio para realizar investigaciones, redactar de correos electrónicos, blogs, entre otros- y de procesadores de texto. Esto exige a los estudiantes el desarrollo de nuevas destrezas de pensamiento crítico (Baquero \& Correa, 2017; Solé, 2009), que les permitirán contrastar información en pro de establecer ideas renovadas que aporten a su quehacer.

\section{Función de las TIC}

La sociedad de la información digital asombra más frecuentemente con artefactos tecnológicos que se hacen parte del ser humano en todo ámbito. Labor de la educación es mantenerse alerta a esta realidad, pues ella asume la responsabilidad de proveer a los alumnos de las habilidades necesarias que requerirán en el futuro.

Las tecnologías de información y comunicación, TIC, se desarrollan como un medio de expresión y creación multimedia, poniendo a disposición múltiples recursos de transmisión de información (Tadeu, 2017); actúan como un canal de comunicación que permite el intercambio de ideas; posibilitan el procesamiento de información y su almacenaje. Son una fuente abierta de documentación. Además, brindan apoyo a los procesos mentales de las personas, constituyéndose en una herramienta de gestión administrativa. 
También, cumplen una función recreativa y son un medio didáctico efectivo que reduce tiempos y facilita el desempeño cognitivo.

Cuando se incorporan las tecnologías al currículo se requiere establecer estrategias adecuadas que se orienten en la labor de generar aprendizaje.

Desde este punto de vista, se entiende la importancia de la incorporación tecnológica con un objetivo pedagógico establecido de manera previa.

\section{Las TIC y la lectoescritura}

Reconociendo que el uso de las TIC ya está instalado en nuestra sociedad, la pregunta es ¿de qué forma encauzamos su utilización de manera que constituya un aporte en el trabajo pedagógico de enseñanza aprendizaje de la lectoescritura?

Dado que la utilización de e-mails, blogs, mensajes de textos, entre otros, es una realidad, es viable pensar que los niños y niñas deben incorporarlo en su tarea educativa $y$, por tanto, en el proceso de adquisición de la lectoescritura.

Sabemos que las TIC son herramientas muy atractivas para los estudiantes y, si se considera que estas tecnologías están hoy inmersas en la sociedad, hacer uso de estos recursos en la labor educativa se convierte más que en una opción, en un deber: formar a los estudiantes con aquellas habilidades que les permitirán desenvolverse en el siglo XXI.

La adquisición de la lectoescritura es un factor decisivo en los logros académicos y en la participación ciudadana (Villalón, 2008). Además, la Organización de las Naciones Unidas para la Educación, la Ciencia y la Cultura (Unesco, 20I8), le otorga una gran importancia a las TIC, por las transformaciones que pueden ejercer en la educación; por lo tanto, el uso de las tecnologías es un predictor positivo en el aprendizaje de la lectoescritura.

El aprendizaje de la lectoescritura es el resultado de un proceso cognitivo (Borrero, 2008; Wolf, 2008), que se inicia mucho antes de 
que los estudiantes lleguen a primero básico. La facilidad o dificultad con que se adquiere depende de cómo es el proceso evolutivo de las habilidades verbales; por otra parte, el uso de las tecnologías también es habitual mucho antes de que los niños y niñas inicien su etapa escolar. La idea es internalizar la lectoescritura como un medio para entender el mundo y expresarse (Tedesco, 2017).

La acción que ejercen las TIC en la adquisición de la lectoescritura, cuando son mediadas adecuadamente por el docente, puede enriquecer y actuar positivamente en el proceso educativo (Toro, 2017). Actualmente son múltiples las alternativas de utilización: programas, software y diferentes recursos a los que se puede acceder. Estos promueven un clima de aula eficiente, que facilita a los estudiantes acercarse al mundo de las letras de manera lúdica y diferenciada, permitiendo el acceso a múltiples alternativas de aprendizaje. Se sabe que todos aprendemos de distinta forma; las TIC abren un nuevo camino de enseñanza-aprendizaje cuando son incorporadas de manera sistemática y planificada (Fernández, 2017; Unesco, 20II; 2018).

El objetivo de la investigación fue determinar la influencia ejercida por las TIC en el desarrollo del proceso de adquisición de la lectoescritura, con el fin de enunciar ventajas y desventajas de su incorporación a este proceso.

\section{Supuestos que guiaron la investigación}

I. La integración de las tecnologías de información y comunicación en el proceso de enseñanza aprendizaje de la lectoescritura contribuye a la obtención de mejores resultados.

2. Las TIC en el aula favorecen la adquisición de la lectoescritura en un tiempo menor.

3. El nivel de motivación de los aprendices es mayor cuando se integran las TIC a la didáctica educativa.

4. Es fundamental la disposición y capacitación del docente para la incorporación de las TIC al aprendizaje.

5. Los alumnos adquieren mayor autonomía y otorgan mayor significado a su aprendizaje cuando se incorporán las TIC. 


\section{Método}

\section{Población y muestra}

Esta investigación fue realizada en el Colegio Mayor Tobalaba, ubicado en la comuna de Puente Alto de la Región Metropolitana de Chile, en el nivel de primeros básicos.

La población estuvo constituida por 60 estudiantes de primer año básico, de un colegio particular pagado de nivel socioeconómico medio-alto, cuyas edades fluctuaban entre los seis y siete años. Se tomó como muestra dos cursos: el primer año básico A, con 17 niñas y I3 niños, y el primer año básico C, con I4 mujeres y I6 varones.

Tabla I: Datos de la muestra.

\begin{tabular}{|l|c|c|c|}
\hline \multicolumn{1}{|c|}{ CURSO } & MUJERES & VARONES & TOTAL \\
\hline Primero A & 17 & I3 & 30 \\
\hline Primero C & I4 & I6 & 30 \\
\hline
\end{tabular}

Fuente: elaboración propia.

La muestra representa a dos de los tres primeros básicos de un universo de I.050 estudiantes. Los estudiantes que asisten el primer año básico A pertenecen al establecimiento educativo desde educación preescolar en un $87 \%$; por su parte, un $90 \%$ de los alumnos de primer año $C$ se integran por primera vez al Colegio en el nivel de Kinder.

\section{Instrumentos}

Se seleccionaron aquellos instrumentos que permitieran recabar información valiosa para la investigación. Por esta razón, se determinó realizar notas de campo, aproximándose a la realidad; también se elaboraron registros para comparar y analizar la información recogida.

Otro medio utilizado fue la entrevista: "desde el punto de vista del método, es una forma específica de interacción social que tiene por objeto recolectar datos para una investigación”" (Sabino, I992, p. II6).

El tercer instrumento utilizado fue la encuesta, que permitió indagar sobre el uso de las TIC por parte de los alumnos. 


\section{Procedimiento de recogida y análisis de datos}

Notas de campo: Con el fin de legitimar cualitativamente el uso de las TIC en la sala de clases, se realizaron notas de campo en los cursos primero A y primero C durante $\mathrm{I} 0$ días, en sesiones de 45 minutos, en clases de lenguaje y en laboratorio de computación, en horario de tecnología. Las observaciones fueron no participantes, complementadas con grabaciones de audio y video. El objetivo fue recoger información explícita respecto del uso de las TIC para un posterior análisis.

Cada observación fue seguida de un registro, con un detallado informe respecto de la hora, fecha, día y el tipo de indagación. Se usaron recursos de grabación de audio y video y observación directa. Esto hizo posible "palpar" el contexto en cada uno de los casos.

Entrevistas: Este instrumento fue una instancia que permitió conversar e intercambiar información entre las entrevistadoras y cinco docentes de primer ciclo básico del establecimiento educacional, cuya experiencia laboral fluctuaba entre los 7 y los 20 años. El instrumento incluyó preguntas generales respecto del uso de la tecnología en el proceso de adquisición de la lectoescritura y otras relacionadas a cómo las aplicaban en el aula. Su objetivo apuntó a establecer la influencia ejercida por las TIC en el desarrollo del proceso de lectoescritura.

Encuesta: Con el fin de recoger el sentir de alumnos y alumnas respecto de la utilización de las TIC en el trabajo en sala de clases, se realizó un cuestionario orientado a conocer la percepción de los estudiantes sobre el uso de las tecnologías de información y comunicación.

Se encuestó a 60 estudiantes de primer año básico. El instrumento incluyó un primer ítem de preguntas cerradas con alternativas de respuesta. El estudiante debía escoger aquella opción que más lo representaba. En un segundo ítem se planteó una pregunta abierta para que explicitaran cuál era la herramienta TIC de su preferencia y por qué.

\section{Resultados}

De la información recogida en las notas de campo, se puede concluir que el uso de tecnologías es una importante fuente de motivación 
para los niños, porque genera un clima potenciador del proceso de enseñanza aprendizaje. Esta apreciación se condice con los resultados obtenidos en una investigación realizada en la Red Iberoamericana, que aplicó las TIC en niños con discapacidad visual como apoyo al aprendizaje de la lectoescritura (Red Iberoamericana de Expertos en la Convención sobre los Derechos de las Personas con Discapacidad, CDPD, 2017), y otra que destaca la uitlización de las tecnologías como un elemento motivador (Fernández-Cruz, Fernández-Díaz, 2016; García, Basilotta \& López, 2014).

Extraemos del texto: El Placer de usar TIC en el Aula Infantil: "Nuestros alumnos están impregnados de una cultura tecnológica que provoca cambios importantes en la manera de aprender y procesar la información" (Asorey \& Gil, 2009, p. I I2); por lo tanto, el uso de las tecnologías deben estar insertas dentro de la sala de clases para enseñar a pensar a los estudiantes.

Otro resultado que se desprende de las notas de campo es que el interés personal del docente por incluir la tecnología en el aula constituye un componente importante, dado que incide en su práctica pedagógica. Esto se concluye luego de las observaciones realizadas a los dos cursos de primero básico de una misma institución, con docentes que han asistido al mismo tipo de capacitaciones $y$, sin embargo, no hacen uso de recursos tecnológicos en la misma medida. Dotar al docente de recursos y capacitarlo en su uso parece no ser suficiente; es necesario hacerlo partícipe de la integración curricular de las TIC para que su puesta en práctica sea realmente efectiva.

Respecto de las entrevistas, atendiendo a los resultados, se puede concluir que el uso de las TIC en el trabajo educativo es una preocupación consciente; tanto por parte de los docentes como de la institución educativa.

Se presenta a continuación los resultados de la encuesta realizada a los estudiantes. 
Tabla 2: Uso del computador en la sala de clases por cursos.

\begin{tabular}{|l|c|c|}
\hline \multicolumn{1}{|c|}{ PREGUNTA N ${ }^{\circ} \mathrm{I}$} & $\mathrm{I}^{\circ} \mathrm{A}$ & $\mathrm{I}^{\circ} \mathrm{C}$ \\
\hline Sí & $66,6 \%$ & $97 \%$ \\
\hline No & $0 \%$ & $0 \%$ \\
\hline No lo hemos usado & $33,4 \%$ & $3 \%$ \\
\hline
\end{tabular}

Fuente: elaboración propia.

Respecto de la pregunta: ¿te gusta que se use computador en el aula?

En la tabla 2 se observa que en primero A la gran mayoría de los niños y niñas disfrutan el uso del computador en sala; sin embargo, un $33,4 \%$ señala no haberlo utilizado. Se destaca que la alternativa "no me gusta" no es seleccionada por ningún estudiante de ese nivel. En tanto en el primero C un $97 \%$ de los alumnos y alumnas registran agrado frente al uso del computador y solo un 3\% declara no haberlo usado.

Tabla 3: Uso de recursos audiovisuales.

\begin{tabular}{|l|c|c|}
\hline \multicolumn{1}{|c|}{ PREGUNTA N ${ }^{\circ}$} & $\mathrm{I}^{\circ} \mathrm{A}$ & $\mathrm{I}^{\circ} \mathrm{C}$ \\
\hline Sí & $83,3 \%$ & $97 \%$ \\
\hline No & $6,7 \%$ & $0 \%$ \\
\hline No lo hemos usado & $\mathrm{I} \% \%$ & $3 \%$ \\
\hline
\end{tabular}

Fuente: elaboración propia.

\section{En cuanto a la pregunta 2: ¿te gusta ver videos en la presentación de letras?}

De acuerdo con la tabla 3 se puede concluir que en ambos cursos los estudiantes muestran un claro interés por la inclusión de actividades audiovisuales en sus trabajos en aula.

Tabla 4: Percepción de los estudiantes.

\begin{tabular}{|l|c|c|}
\hline \multicolumn{1}{|c|}{ PREGUNTA N $^{\circ} 3$} & $\mathrm{I}^{\circ} \mathrm{A}$ & $\mathrm{I}^{\circ} \mathrm{C}$ \\
\hline Sí & $86,7 \%$ & $3 \%$ \\
\hline No & $10 \%$ & $97 \%$ \\
\hline No lo hemos usado & $3 \%$ & $0 \%$ \\
\hline
\end{tabular}

Fuente: elaboración propia. 


\section{En la pregunta 3: ¿son entretenidas las clases con pizarra y plumón?}

La tabla 4 refleja la percepción que tienen los niños y niñas respecto del uso de pizarra y plumón en clases de lectoescritura. En el curso $\mathrm{I}^{\circ} \mathrm{A}$ los estudiantes mantienen una sensación positiva respecto de sus clases (86,7\%). Recordemos que no está en cuestionamiento la efectividad de las diversas estrategias utilizadas en el campo educativo; lo que la investigación intenta detectar es el impacto positivo de la incorporación de las TIC al aula, y la tabla 4 muestra que éstas resultan atractivas en un $97 \%$ en el curso $\mathrm{I}^{\circ} \mathrm{C}$, donde sí se incorporan.

Tabla 5: Percepción de los estudiantes del trabajo en aula.

\begin{tabular}{|l|c|c|}
\hline \multicolumn{1}{|c|}{ PREGUNTA N ${ }^{\circ}$} & $\mathrm{I}^{\circ} \mathrm{A}$ & $\mathrm{I}^{\circ} \mathrm{C}$ \\
\hline Sí & $63,3 \%$ & $97 \%$ \\
\hline No & $33 \%$ & $0 \%$ \\
\hline No lo hemos usado & $3,3 \%$ & $3 \%$ \\
\hline
\end{tabular}

Fuente: elaboración propia.

En relación con la pregunta 4 , ¿te gusta trabajar con actividades de libro proyectadas en la pizarra?, la tabla 5 muestra claramente la preferencia por la inclusión de actividades en la que se usan proyectores y se visualiza las actividades de libro.

Tabla 6: Interés manifestado por los estudiantes.

\begin{tabular}{|l|c|c|}
\hline \multicolumn{1}{|c|}{ PREGUNTA No5 } & $\mathrm{I}^{\circ} \mathrm{A}$ & $\mathrm{I}^{\circ} \mathrm{C}$ \\
\hline Sí & $80 \%$ & $97 \%$ \\
\hline No & $7 \%$ & $0 \%$ \\
\hline No lo hemos usado & $13 \%$ & $3 \%$ \\
\hline
\end{tabular}

Fuente: Elaboración propia

En cuanto a la pregunta 5, ¿Te gusta trabajar con pantalla digital?, la tabla 6 evidencia el interés de los estudiantes por participar en actividades tecnológicas. Se observa también que aquellos niños y niñas que están más expuestos a las TIC tienen mayor interés por acceder al uso y práctica de herramientas de este tipo. 
Tabla 7: Trabajo en laboratorio tecnológico.

\begin{tabular}{|l|c|c|}
\hline \multicolumn{1}{|c|}{ PREGUNTA N ${ }^{\circ} 6$} & $\mathrm{I}^{\circ} \mathrm{A}$ & $\mathrm{I}^{\circ} \mathrm{C}$ \\
\hline Sí & $90 \%$ & $\mathrm{I} 00 \%$ \\
\hline No & $0 \%$ & $0 \%$ \\
\hline No lo hemos usado & $\mathrm{I} \% \%$ & $0 \%$ \\
\hline
\end{tabular}

Fuente: elaboración propia.

Con relación a la pregunta: ¿disfrutas de las actividades en el laboratorio de computación?, la tabla 7 muestra que todos los alumnos del primero $\mathrm{C}$ que han participado en laboratorios de computación tienen una percepción positiva del trabajo con esta herramienta. Sin embargo, los resultados obtenidos en el primero $\mathrm{C}$ son muchos más homogéneos que en el primero A. En las preguntas I a 4 el curso primero $\mathrm{C}$ obtuvo los mismos resultados positivos y negativos; en la pregunta 5, en cambio, la apreciación de estos alumnos fue un $100 \%$ positiva.

\section{Conclusiones}

En la sociedad de la información es evidente la necesidad de incorporar el uso de las TIC al trabajo educativo (Cassany, 2008). Las generaciones actuales han nacido en la era de las tecnologías, por tanto, no conciben una realidad sin ellas. Los profesores deben capacitarse para utilizar estas herramientas con un fin pedagógico, por los aspectos positivos que generan en el aprendizaje, entre ellos, motivación constante (Toro, 2017), autoestima, capacidad de innovación, características que son resultados de varias investigaciones (Colón, et al., 20I4; Suárez, et al., 20I5; ADCP, 2017; Porras \& Pérez, 2017).

E1 Ministerio de Educación en Chile (2012), en sus bases curriculares, propone de manera explícita la integración de las TIC en todas las asignaturas, con el propósito de trabajar con información, desarrollando una actividad reflexiva y pensamiento crítico para seleccionar aquello que resulta importante. Permiten, además, establecer vínculos de comunicación con personas de diversos lugares y culturas. La utilización de las TIC en un trabajo planificado 
constituye una importante herramienta de aprendizaje. Esto requiere el uso responsable de recursos tecnológicos por parte de la escuela.

La investigación desarrollada permitió llegar a las siguientes conclusiones:

La utilización de las TIC produce en los niños un "mágico encanto" que seduce la natural y espontánea curiosidad que acompaña su crecimiento. Hoy por hoy, para los docentes, valerse de este recurso va más allá de una opción, se constituye en una obligación si se piensa que los estudiantes crecen en una sociedad digital y deben desarrollar habilidades para el siglo XXI.

La investigación fue realizada en un colegio particular pagado de una comuna de la Región Metropolitana de Santiago de Chile. Esta institución cuenta con un programa de integración de tecnología. En él, los docentes acceden a los recursos y capacitaciones requeridas. Los estudiantes hacen uso de las TIC en el centro educativo, además de poder acceder a estas herramientas en sus hogares.

En las entrevistas realizadas a las profesoras se destaca como ventaja del uso de las TIC la motivación e interés que promueven en las clases de lenguaje, reportándose un aumento en tiempos de atención y concentración por parte del alumnado. Similares resultados se obtienen de las encuestas realizadas a los estudiantes, las cuales muestran un elevado interés por la incorporación de herramientas tecnológicas en actividades escolares.

Por otro lado, las notas de campo dan indicios de las dificultades que se presentan al pretender incorporar las TIC en actividades académicas. Se observó a dos cursos de la misma institución, que tienen la misma oportunidad de acceder a los recursos de TIC; sin embargo, por falta de interés o motivación, una de las profesoras no hace uso de este tipo de herramientas en el desarrollo de las actividades de lectoescritura.

Por consiguiente, se puede establecer que una desventaja es que no solo depende de que el docente cuente con la capacitación adecuada cuando se busca incorporar las TIC, se necesita además que el docente se empodere de su utilización. 
Los niños y niñas están dispuestos a la incorporación de tecnología en su trabajo escolar, porque, por un lado, no solo les nace la inquietud por aprender, sino que también le favorece la incorporación a una sociedad tecnificada (Fernández, 2017); por otra parte, les desarrolla la capacidad de abrir sus mentes para un aprendizaje más dinámico y con menos tiempo de inversión en las tareas escolares (CDPD, 2017).

Es labor del profesor mediar el uso de TIC hacia el proceso de lectoescritura. Él debe buscar estrategias para que los alumnos aprendan de manera distinta. Para ello, las TIC se convierten en un importante propulsor de aprendizaje; junto con la influencia del profesor y de la familia, son agentes esenciales en el gusto hacia la lectura (Fernández, 2017; Paredes, 2005).

Durante la investigación se observaron las clases de dos primeros básicos, en los cuales se implementó el método integrado como metodología de enseñanza de la lectoescritura. En uno de ellos la docente a cargo incorporaba el uso de TIC a su didáctica, en contraste a lo observado en el otro curso, en el cual no se evidenciaba el uso de los recursos tecnológicos. Al revisar los porcentajes de logro y calificaciones de ambos cursos, es posible detectar que el primero básico que hace uso de TIC alcanza mejores resultados. Esto podría ser atribuido a la utilización de los recursos tecnológicos.

Las notas de campo dan cuenta de que los estudiantes que asisten al primer año básico $\mathrm{C}$ se observan más motivados e interesados en el trabajo que se desarrolla en clases.

Las nuevas tecnologías permiten revisar los contenidos de manera lúdica e interactiva. Bajo este prisma, existen múltiples y variados recursos en red que promueven la práctica y aprendizaje de la lectoescritura; está en manos del docente hacer uso adecuado y oportuno, previendo una planificación consciente de los requerimientos necesarios para su éxito.

A continuación, se enumeran ventajas y desventajas en el uso de las TIC derivadas de la investigación. 


\section{Ventajas}

- Motivación: ejerciendo un importante estímulo hacia el aprendizaje.

- Aprendizaje significativo: al apelar a lo lúdico, el contenido es más revelador para los estudiantes.

- Atención y concentración: el atractivo que genera desencadena un aumento en el nivel y en la prolongación en tiempo del interés del contenido trabajado.

- Autoaprendizaje: permite el trabajo personal.

- Respeto a los ritmos de aprendizaje: asociado a la ventaja antes mencionada, permite que el estudiante vaya avanzando según sus propios tiempos de aprendizaje, facilitando la práctica y el ejercicio.

- Considera inteligencias múltiples: al abarcar la utilización de las TIC la estimulación de los diversos sentidos, facilita el desarrollo de las inteligencias múltiples.

- Capacita en el nuevo concepto de alfabetización: la gran cantidad de posibilidades que implica su uso, especialmente el acceso a internet, permite familiarizarse con diversos textos e hipertextos, introduciéndose a un concepto moderno.

- Promueve la creatividad: dada la gama de posibilidades a las que se accede.

- Promueve la equidad de acceso a la educación: facilita el acceso a toda información, ampliando las posibilidades de comunicación y conocimientos.

\section{Desventajas}

- Depende de la capacitación e interés del docente: el correcto y adecuado uso de las TIC es muy sensible a la disposición que manifieste el docente.

- Existe gran riesgo de dispersión: si no existe una planificación adecuada, es muy posible que se pierda el objetivo al que se aspira, tanto por parte del docente como del alumnado. 
- Dependiente de recursos económicos: su implementación resulta costosa.

- Factores técnicos influyen en la fluidez de las clases: dificultades ajenas a las posibilidades del docente pueden influir en la correcta puesta en práctica; por ejemplo, problemas de conectividad.

- Requiere de especial cuidado del docente para que no exista abuso de la tecnología, perdiéndose la adecuada mediación: el docente debe estar atento a realizar la mediación oportuna en el trabajo que realizan los estudiantes en las TIC.

- Aumenta la brecha digital: por tratarse de un recurso de alto costo, los estudiantes que no participan de una institución que cuente con estos recursos se ven limitados en términos de acceso a conocimientos y capacitación.

\section{Discusión}

Es la institución educativa la que debe proveer de los recursos tecnológicos para que el profesor pueda hacer uso de ellos al interior del aula (García-Rodríguez y Gómez Díaz, 2017); también es necesario que la organización entregue la capacitación en el uso de las TIC (Fernández-Cruz, Fernández-Díaz, 2016) relacionado con procesos de lectoescritura; de lo contrario, es muy difícil la correcta implementación sin el apoyo y alineación de los directivos, profesores y la escuela.

\section{Referencias bibliográficas}

Asorey, E. y Gil, J. (2009). El placer de usar las Tics en el aula infantil. CEE Participación Educativa, 12, IIO-II9.

Baquero, L. y Correa, D. (2017). Transformación de las prácticas de aula en la escuela para la construcción del hábito lector. Hexágono Pedagógico, 8(I), 49-62. DOI 10.22519/2I45888X.1060.

Borrero, L. (2008). Enseñando a leer: Teoría, práctica e intervención. Bogotá: Editorial Norma.

Cassany, D. (2008). Prácticas letradas contemporáneas. Ciudad de México: Ríos de Tinta. 
CDPD. (2017). Utilización de las TIC por parte de los alumnos con discapacidad visual como elemento de apoyo al aprendizaje de la lectoescritura. Revista sobre discapacidad visual, 62, I-I7. Recuperado de http://www.once.es/ new/servicios-especializados-en-discapacidad-visual/publicacionessobre-discapacidad-visual/nueva-estructura-revista-integracion

Colón, A. O., Moreno, L.A., León, M.P. y Zagalaz, J.C. (2014). Formación en TIC de futuros maestros desde el análisis de la práctica en la Universidad de Jaén. Píxel-Bit, 44, I27-I42. Recuperado de http://-goo.gl/g6WSRy

Falieres, N. (2006). Cómo enseñar con las nuevas tecnologías en la escuela de boy. Buenos Aires: Círculo Latino Austral.

Fernández, J. (2017). Tecnologías de la Información y Comunicación (TIC) y Educación Inclusiva. Un Pacto de Futuro. En El Homrani, M. (coord.), Entornos y Estrategias Educativas para la Inclusión Social (pp. 57-68). Granada: Comares.

Fernández-Cruz, F. y Fernández-Díaz, M. (2016). Los Docentes de la Generación Z y sus competencias digitales. Revista Comunicar, 46(24), 97-I05. DOI http://dx.doi.org/I0.3916/C46-2016-I0

García-Rodríguez, A. y Gómez-Díaz, R. (2017). Lectura digital infantil y juvenil en tabletas y smartphones: Una oportunidad para lograr Buenos lectores. Recuperado de https://listserv.rediris.es/cgi-bin/ wa?A2=indI70IE\&L=IWET $\mathrm{EL} \& \mathrm{~F}=2 \mathrm{~S}=\& \mathrm{P}=48 \mathrm{I} 2$.

García, A., Basilotta, V. y López, C. (20I4). Las TIC en el aprendizaje colaborativo en el aula de primaria y secundaria. Comunicar, Revista Científica de Educomunicación, 2l(42), 65-74.

Paredes, J. (2005). Animación a la lectura y TIC, creando situaciones y espacios. Revista de Educación, número extraordinario, 255-279. Recuperado de https://www.biblioposes/animación-a-la-lectura-y-tic-creandosituaciones-y-espacios/

Porras C. y Pérez J. (2017). Implementación de las TIC como estrategia de apoyo al proceso de lectoescritura. Caso de aplicación: Institución Educativa Maipore, sede A, Bucaramanga. Textos y Sentidos. Recuperado de http://biblioteca.ucp.edu.co/ojs/index.php/textosysentidos/ article/view/3255

Sabino, C. (1992). El proceso de investigación. Caracas: Ed. Panapo.

Sáez, J. (2010). Utilización de las TICs en el proceso de enseñanza aprendizaje, valorando la incidencia real de las tecnologías en la práctica docente. Revista Docencia e Investigación, 20, I83-203.

Solé, I. (2009). Estrategias de lectura. Barcelona: Graó. 
Suárez, A., Pérez C., Vergara, M. y Alférez V. (2015). Desarrollo de la lectoescritura mediante TIC y recursos eucativos abiertos. Revista de Educación Innovativa, 7(I), 38-49.

Tadeu, P. (2017). La inclusión educativa social desde una perspetciva portuguesa, en El Homrani, M. (coord.), Entornos y Estrategias Educativas para la Inclusión Social (pp. 327-338). Granada: Comares.

Tedesco, J. C. (2017). Educación y desigualdad en América Latina y el Caribe. Aportes para la agenda post 2015. Perfiles educativos, 39(I58), 206-224. Recuperado de http://www.scielo.org.mx/scielo.php?script $=$ sci_ arttext\&pid=S0185-26982017000400206\&lng=es\&tlng=es.

Toro, C. (2017). Las TIC: Estrategia para mejorar la competencia lectorainterpretativa en el área de lenguaje. Revista Universidad Católica Luis Amigó, l, I26-I48.

Unesco (20I I). Unesco ICT Competency Framework for Teachers. Recuperado el 8 de octubre de 2018 de http://goo.gl/oKUkB.

Unesco (2018). Las TIC en Educación. Recuperado de https://es.unesco.org/ themes/tic-educacion

Villalón, M. (2008). Alfabetización inicial. Santiago: Ediciones Universidad Católica de Chile.

Wolf, M. (2008). Cómo aprendemos a leer. Barcelona: Ediciones B.S.A. 